\title{
The ecology of terrestrial eDNA: Its state, transport, and fate on aboveground surfaces
}

\author{
Rafael Valentin ${ }^{1}$, Kathleen Kyle ${ }^{2}$, Michael Allen ${ }^{2}$, Dustin Welbourne ${ }^{3}$, and Julie Lockwood ${ }^{2}$ \\ ${ }^{1}$ Princeton University \\ ${ }^{2}$ Rutgers University \\ ${ }^{3}$ University of Florida
}

May 5, 2020

\begin{abstract}
Environmental DNA (eDNA) has become a valuable tool for monitoring species of concern or site biodiversity, including expanded use of surveys designed to detect fully terrestrial species. However, if aboveground terrestrial eDNA surveys are to be widely adopted, it is necessary to first understand how terrestrial conditions affect the state, transport, and ultimate fate (or 'ecology') of terrestrially deposited eDNA. Many of the processes that affect eDNA's state, transport, and fate in aquatic environments may not be applicable in aboveground systems, warranting an exploration of the terrestrial processes that likely do affect eDNA. Here we explore ecology terrestrial eDNA through a series of experiments exploring the optimal filter pore size for eDNA collection, how eDNA is affected by rain events, and its degradation rate under different solar radiation conditions. We found that the capture concentration of intracellular eDNA was not significantly affected by an increase in filter pore size, suggesting there is a wide range of viable pore size options for targeting intracellular eDNA. We also found extracellular eDNA degrades more rapidly than intracellular forms when exposed to solar radiation, indicating the latter is a more viable target for collection. Finally, we identified that rainfall or mist will remove most terrestrial eDNA present on vegetation substrate. This study provides researchers and managers key insights into successfully designing and carrying out terrestrial eDNA surveys that maximize detection probability and reduce production of false positive survey results.
\end{abstract}

\section{Hosted file}

Valentin et al., 2020 - The ecology of terrestrial eDNA.pdf available at https://authorea. com/users/304054/articles/434428-the-ecology-of-terrestrial-edna-its-state-transportand-fate-on-aboveground-surfaces 